\title{
Microbial Correlates of Fusarium Load and Deoxynivalenol Content in Individual Wheat Kernels
}

\author{
Matthew G. Bakker† and Susan P. McCormick \\ United States Department of Agriculture-Agricultural Research Service, Mycotoxin Prevention \& Applied Microbiology, Peoria, IL 61604 \\ Accepted for publication 31 January 2019.
}

\begin{abstract}
Characteristics or constituents of plant-associated microbiomes may assist in constraining disease development. To investigate this possibility for the wheat-Fusarium head blight pathosystem, we assessed seed weight, pathogen load, deoxynivalenol content, and microbiome profiles for individual wheat kernels collected over 2 years from a disease-conducive environment. We found that the microbiomes of individual, hulled wheat kernels consist of dozens to greater than a hundred bacterial taxa and up to several dozen fungal taxa, and that year-to-year variation in microbiome structure was large.

measures of disease severity, and had significant power to explain variation in pathogen load among seeds. Several operational taxonomic units belonging to the genus Sphingomonas demonstrated particularly strong negative relationships with pathogen load. This study illuminates the composition of microbiomes associated with wheat kernels under disease-conducive field conditions, and suggests relationships between microbiome characteristics and Fusarium head blight that warrant further study.
\end{abstract} Measures of microbial community diversity were negatively correlated with

Fusarium head blight is a devastating disease of small grains, regularly imposing economic costs in the billions of dollars (Nganje et al. 2001) and substantially impacting where small grains can be profitably grown (Windels 2000). Costs associated with Fusarium head blight are two-fold: yield losses due to failure to set seed and reduced seed size, and severe price discounts associated with the presence of mycotoxins in infected grain. The latter arises because grain contaminated with mycotoxins at concentrations above tolerable limits must be diverted out of the food and feed supply as a result of toxicity to people and animals (Bulder et al. 2011).

Research is needed to better understand how the Fusarium head blight pathogens (in North America, predominantly Fusarium graminearum and its close relatives) (Starkey et al. 2007) interact with the broader community of microorganisms that inhabit the same plant tissues (i.e., the microbiome, of which the pathogen is a component part). The presence and activity of other members of the microbiome may influence Fusarium head blight progression at various points, including the effectiveness with which the pathogen establishes on the head, the rate at which the pathogen spreads among florets, the total distance of such secondary spread, the density of pathogen biomass that is achieved, or the production and accumulation of mycotoxins.

Some evidence of the potential for microbiome interactions to influence Fusarium head blight progression can be seen in the counterintuitive effect by which application of certain fungicides belonging to the quinone outside inhibitor group actually increases Fusarium success and the accumulation of mycotoxins (Simpson et al. 2001); disruption of the microbiome may, in some cases,

${ }^{\dagger}$ Corresponding author: M. G. Bakker; E-mail: Matt.Bakker@ars.usda.gov

Funding: This work was supported by the United States Department of Agriculture-Agricultural Research Service National Program for Food Safety.

*The $\boldsymbol{e}$-Xtra logo stands for "electronic extra" and indicates that three supplementary figures and three supplementary materials are published online.

The author(s) declare no conflict of interest.

This article is in the public domain and not copyrightable. It may be freely reprinted with customary crediting of the source. The American Phytopathological Society, 2019.
Keywords: biological control, ecology and epidemiology, mycology.

alleviate natural pathogen suppression, permitting pathogen proliferation. With sufficient understanding of community dynamics and species interactions within the microbiome, novel disease control approaches based on microbiome management may become possible (Bakker et al. 2012; Kinkel et al. 2012).

Study of pathogen-microbiome interactions may also yield promising new candidates for inoculative biological control; other microbial taxa that are consistently associated with poor pathogen performance, even under environmentally disease-conducive conditions, may be developed into disease control technologies for crop production. There have been efforts to develop biological control products for Fusarium head blight (Hue et al. 2009; Jung et al. 2013; Legrand et al. 2017; Schisler et al. 2015) but additional discovery efforts in this area remain vital.

Several features of the Fusarium head blight pathosystem lend themselves to a biological control approach. For instance, initial infection and pathogen establishment are generally thought to occur at anthesis, and weather-related variables within a few days of anthesis can reliably model Fusarium head blight risk (Shah et al. 2013). Thus, this system may not require strong control over an entire growing season, which can be difficult to obtain with biologicals. It should be noted, however, that infections at later growth stages are also possible (Del Ponte et al. 2007; Siou et al. 2014). Furthermore, because grain fill and physiological maturity are reached rapidly after flowering in the small grains, the time interval between vulnerability to infection and harvest of grain is short. This can limit options for chemical control because of minimum preharvest intervals for some products.

Several recent studies have profiled microbiomes associated with the phyllosphere of small grain crops (Gdanetz and Trail 2017; Karlsson et al. 2014; Nicolaisen et al. 2014), complementing cultivation-based studies that have a longer history (Comby et al. 2016; Larran et al. 2002, 2007; Legard et al. 1994). However, these studies have not always sampled plants in disease-conducive environments, which limits their applicability to exploring microbial interactions that may constrain Fusarium head blight damage. Furthermore, for specific insights into Fusarium head blight progression, microbiome profiles of tissues directly involved in the disease will be most relevant. For instance, some microbial taxa associated with the wheat phyllosphere are recovered at much different frequencies when sampling is directed at stems versus 
grains (Grudzinska-Sterno et al. 2016) or kernels versus rachis versus anthers (Comby et al. 2016).

In this work, we sampled individual hulled wheat kernels from a mist-irrigated field nursery over 2 years. We assessed kernel weight, mycotoxin content (deoxynivalenol [DON]), pathogen load, and microbiome profiles. Our objective was to test whether placing pathogen success in the context of broader microbiome characteristics could provide new insight into the development of Fusarium head blight and the accumulation of mycotoxins in grain. Our hypothesis was that Fusarium head blight severity is significantly related to microbiome characteristics and to the abundances of particular members of the wheat kernel microbiome, which may represent novel management targets.

\section{MATERIALS AND METHODS}

Generation of infected wheat kernels in a field setting. Wheat kernels were collected over two seasons (2016 and 2017) from a pathogen-inoculated, mist-irrigated nursery for Fusarium head blight screening at the Experiment Station of the University of Minnesota (St. Paul, MN, U.S.A.; 44.99 N, 93.18 W). Field soil at this location is a Waukegan silt loam. The previous crop was soybean and the field was prepared with chisel plowing. Planting occurred on 20 May 2016 and 15 May 2017. Seeds were planted in a randomized complete block design with three replicates, using single-row plots of various cultivated wheat lines and landraces. Row spacing was $17.75 \mathrm{~cm}$ and planting density was $2.5 \times 10^{6}$ seeds $\mathrm{ha}^{-1}$. Fertilization followed standard recommendations for commercial wheat production in Minnesota. No fungicides were applied, either to seed before planting or as foliar applications.

The field plot was inoculated with a mixture of $F$. graminearum isolates (30 isolates in 2016 and 40 in 2017; all originally collected from wheat fields and barley fields in Minnesota), which spanned a range in aggressiveness and capacity for producing the mycotoxin DON. To produce inoculum, each isolate (maintained individually in soil) (Windels et al. 1993) was transferred to mung bean agar medium (boil $40 \mathrm{~g}$ of mung beans in 1 liter of filtered water [0.22 $\mu \mathrm{m}$ ] for $23 \mathrm{~min}$; filter through two layers of gauze pads and adjust volume to 1 liter with filtered water; add $15 \mathrm{~g}$ of agar [Difco]; and autoclave on liquid cycle). Isolates were grown under fluorescent and UV lights (cycle of $12 \mathrm{~h}$ of light and $12 \mathrm{~h}$ of darkness) at room temperature $\left(22\right.$ to $\left.24^{\circ} \mathrm{C}\right)$. On the seventh day, $10 \mathrm{ml}$ of water was added to each plate, and the surface was rubbed with a sterile bent-glass rod to loosen macroconidia. The resulting suspension was filtered through two layers of cheesecloth to reduce the number of mycelial fragments, diluted to $40 \mathrm{ml}$, and used to inoculate 20 mung bean agar plates per isolate $(1.5 \mathrm{ml} / \mathrm{plate})$. These plates were incubated as described previously, and macroconidia were harvested by washing plates with approximately $20 \mathrm{ml}$ of deionized water per plate, using a $\mathrm{CO}_{2}$-powered backpack sprayer fitted with a flat-fan spray tip (TeeJet SS8003; Spraying Systems Co.) at an operating pressure of approximately $276 \mathrm{kPa}$. Spores were harvested from an equal number of plates per isolate, and combined. The spore suspension was filtered through two layers of cheesecloth and was adjusted to $8 \times 10^{5}$ spores $\mathrm{ml}^{-1}$, based on direct spore counts using a hemocytometer. Inoculum was stored in 1-liter aliquots at $-80^{\circ} \mathrm{C}$ until use.

On the day of inoculation, stored inoculum was thawed and spore germination was verified by visual observation $8 \mathrm{~h}$ after spreading the spore suspension onto potato dextrose agar. Spore concentration was diluted with water to $1 \times 10^{5}$ spores $\mathrm{ml}^{-1}$ ( 8 liters total volume) and approximately $20 \mathrm{ml}$ of Tween- 20 was added as a wetting agent. Inoculum was dispensed using a $\mathrm{CO}_{2}$-powered backpack sprayer, as described above, at a rate of $30 \mathrm{ml} / \mathrm{m}$ of row. All experimental plots were inoculated at anthesis, and again 3 days later (8 and 11 July 2016 and 6 and 9 July 2017).

Mist irrigation commenced immediately following the first inoculation, in order to prevent overly rapid drying of inoculum. A micro-sprinkle mist-irrigation system (DAN 8000 series with rotating spinner; NETAFIM Irrigation Inc.) was used with risers at a height of $1 \mathrm{~m}$. The mist-irrigation system was programmed to provide 14 misting periods per day $(9 \mathrm{~min}$ each, at hourly intervals starting at 17:00), delivering a daily cumulative total of $10.7 \mathrm{~mm}$ of water.

Samples were collected exclusively from the hard red spring wheat cultivar Rollag, which is commercially produced in Minnesota and is considered to be moderately resistant to Fusarium head blight, carrying the Fhb1 resistance locus (Anderson et al. 2015). Individual heads were sampled 32 days (2016) or 26 days (2017) after the initial inoculation by clipping, bagging, and storing on ice in a cooler in the field. Less than $1 \mathrm{~h}$ after collection, heads were frozen using liquid nitrogen and stored at $-80^{\circ} \mathrm{C}$ prior to express shipment to Illinois on dry ice. One to four kernels were selected arbitrarily per head, removed from the hull using forceps, and placed individually into 1-dram glass vials. Collection of seed from heads aimed to include seed that spanned a range (by visual assessment) of minor to severe symptom expression.

Measurement of DON content of individual kernels. DON was extracted from each kernel with $1.5 \mathrm{ml}$ of acetonitrile-water (86:14) for $24 \mathrm{~h}$ on a vortex mixer. Extract $(1 \mathrm{ml})$ was transferred to a half-dram vial and dried with heat under a stream of nitrogen. Trimethylsilyl (TMS) derivatives were prepared by adding $20 \mu \mathrm{l}$ of a freshly prepared mixture of $\mathrm{N}$-trimethylsilylimadazole and trimethylchlorosilane (100:1; Sigma-Aldrich). After $20 \mathrm{~min}, 180 \mu \mathrm{l}$ of isooctane was added to each sample, followed by $200 \mu \mathrm{l}$ of water. The vials were gently mixed until the top, organic layer became clear. The organic layer was transferred to a $250-\mu 1$ autosampler vial glass insert. TMS derivatives of purified DON were also prepared to construct a standard curve for quantification.

Samples were run through a gas chromatograph (Agilent 7890) fitted with an HP-5MS column $(30 \mathrm{~m}, 0.25 \mathrm{~mm}$, and $0.25 \mu \mathrm{m})$, coupled to a quadrupole mass spectrometer (Agilent 5977). The injection temperature was kept at $250^{\circ} \mathrm{C}$ and the column flow rate was $1 \mathrm{ml} \mathrm{min}-1$. The temperature program held an initial column temperature of $150^{\circ} \mathrm{C}$ for $1 \mathrm{~min}$, then increased to $280^{\circ} \mathrm{C}$ at $30^{\circ} \mathrm{C}$ $\mathrm{min}^{-1}$ and held for $3.5 \mathrm{~min}$. Selective ion monitoring was applied to detect the characteristic ions of DON (derivatized with TMS at three positions) with fragment ion (m/z value) of 235.1 as the target ion and 259.1, 295.1, 392.2, 422.2, and 512.2 as reference ions. The detection limit with this method was $0.025 \mu \mathrm{g}$ of DON.

Measurement of pathogen load in individual kernels. Pathogen load was assessed by quantitative polymerase chain reaction (qPCR). Residual solvent from DON extraction was evaporated, and kernels were lyophilized. Tissue was pulverized by bead beating using a single 3 -mm tungsten-carbide bead in a 2-ml XXTuff Microvial (BioSpec Products) and a Precellys 24 homogenizer (Bertin Technologies) set to 4,200 rpm for $30 \mathrm{~s}$. DNA was extracted from pulverized tissue using the PureLink Plant kit (Thermo Fisher Scientific), according to the manufacturer's directions.

Primers for the trichodiene synthase gene, TRI5 (CuperlovicCulf et al. 2018), were used to estimate the load of mycotoxigenic Fusarium spp. (presumably, primarily the inoculated species, F. graminearum). Pathogen load was expressed relative to wheat DNA content in each extract to avoid biasing estimates according to DNA extraction efficiency. For this purpose, wheat DNA content was measured using primers for the gene PR-1 (Gao et al. 2013). Technical duplicates were run for each sample.

Each reaction mixture consisted of SsoAdvanced Universal SYBR Green supermix (2016; Bio-Rad) or SsoFast EvaGreen master mix (2017; Bio-Rad), forward and reverse primers each at $500 \mathrm{nM}$ final concentration, template DNA, and water (molecular biology grade) to a final volume of $20 \mu \mathrm{l}$. Thermocycling consisted of $98^{\circ} \mathrm{C}$ for $30 \mathrm{~s}, 40$ cycles of $95^{\circ} \mathrm{C}$ for $10 \mathrm{~s}$ and $58^{\circ} \mathrm{C}$ for $30 \mathrm{~s}$, and melt curve from $60^{\circ} \mathrm{C}$ to $95^{\circ} \mathrm{C}$ in $0.5^{\circ} \mathrm{C}$ increments.

Raw fluorescence values at each PCR cycle were exported for processing with the LinRegPCR data analysis program (Ramakers et al. 2003). This analysis method determines the baseline and 
estimates an amplification factor for each sample individually. Starting quantities were calculated with correction for variable amplification efficiencies (for instance, due to variable PCR inhibitor contents), using the formula for estimation of starting concentration (Ruijter et al. 2009) $N_{o}=\left(N_{t} / E^{C t}\right)$, where $N_{o}$ is the starting concentration (in arbitrary fluorescence units), $N_{t}$ is the fluorescence intensity threshold, $E$ is the amplification factor (where $E=2$ indicates a perfect doubling with each PCR cycle), and $C_{t}$ is the cycle number at which the fluorescent signal crosses the fluorescence intensity threshold.

Pathogen load was calculated as Pathogen load $=\ln [(10,000 \times$ $\left.\left.N_{\text {ofusarium }}\right) / N_{\text {owheat }}\right]$, where the multiplier in the numerator was used to prevent the occurrence of negative values after log transformation.

Microbiome profiling in individual kernels. DNA extracted from individual kernels was also used to prepare amplicon libraries for sequencing.

Prokaryote communities were profiled via sequencing amplicons spanning the v5 and v6 regions of the 16S ribosomal RNA (rRNA) gene, using primers 779F (Chelius and Triplett 2001) and the reverse complement of $1114 \mathrm{~F}$ (Lundberg et al. 2012). Fungal communities were profiled via sequencing amplicons of the first internal transcribed spacer region (ITS1), using primers ITS1f and ITS2 (Smith and Peay 2014). Primers were modified with 5' overhangs for compatibility with the MiSeq workflow, and to create a frameshifted mixture of oligos to provide signal diversity within each cycle of sequencing through the primer regions.

Sequencing libraries also included negative control samples (i.e., DNA extractions performed without any kernel tissue and PCRs run without any template DNA) and mock community control samples of known composition. The mock bacterial community was product MSA-1003 from the American Type Culture Collection, while the mock fungal communities were from Bakker (2018).

The reaction mix for amplicon generation consisted of $0.5 \mathrm{U}$ of Phusion High-Fidelity DNA polymerase with associated Phusion Green HF reaction buffer (Thermo Fisher), dNTPs at $200 \mu \mathrm{M}$ final concentration, forward and reverse primers (each at $0.5 \mu \mathrm{M}$ ), $2.5 \mu \mathrm{l}$ of template DNA, and nuclease-free water to a total volume of $25 \mu \mathrm{l}$ per reaction. Thermocycling consisted of $98^{\circ} \mathrm{C}$ for $30 \mathrm{~s} ; 25$ cycles of $98^{\circ} \mathrm{C}$ for $10 \mathrm{~s}, 57^{\circ} \mathrm{C}$ for $30 \mathrm{~s}$, and $72^{\circ} \mathrm{C}$ for $15 \mathrm{~s}$; and final extension at $72^{\circ} \mathrm{C}$ for $5 \mathrm{~min}$. PCR products were cleaned using the SequalPrep Normalization Plate Kit (Thermo Fisher), and 16S and ITS1 amplicons were pooled by sample. An eight-cycle second-round PCR was used to add sample-specific barcode indices, using the Nextera XT Index Kit (Illumina). The manufacturer's protocol was followed, except that we substituted Phusion High-Fidelity DNA polymerase for the suggested polymerase.

Indexed amplicons were cleaned and normalized with the SequalPrep kit ahead of sample pooling. Library quality and concentration were assessed with the TapeStation instrument (Agilent) and with the Library Quantification Kit for Illumina Platforms (Kapa Biosystems). Separate libraries were prepared for kernels from 2016 and from 2017. Sequencing was performed with a MiSeq instrument (Illumina), using version 2 (500-cycle) sequencing kits. Raw sequence data are available in the National Center for Biotechnology Information Sequence Read Archive as accession SRP144973.

Amplicon sequences and resulting taxon abundance tables were processed with Mothur v.1.40.5 (Schloss et al. 2009), ITSx v.1.1 (Bengtsson-Palme et al. 2013), and R v.3.4 (R Core Team 2015). A complete record of commands issued during amplicon sequence processing is provided (Supplementary Material S1).

Briefly, bidirectional reads were merged into contigs. Reads were culled if they contained ambiguous bases, homopolymers longer than 15 bases, or had a total length less than 100 bases. The target 16S rRNA amplicon was retrieved by matching the PCR primers, allowing one mismatch in each of the upstream and downstream primers. Reads were aligned to the Silva reference alignment v. 132
(Quast et al. 2013) and were filtered to a consistent amplicon. Chimeras were identified using VSearch (Rognes et al. 2016), with the Silva database as the reference. Reads differing in two or fewer positions were preclustered. Remaining unique reads were classified using the Silva database and a naïve Bayesian classifier (Wang et al. 2007) as implemented in Mothur, with a confidence threshold of 80; reads that could not be classified below the domain or that were classified as chloroplast or mitochondria were culled. Remaining reads were clustered into operational taxonomic units (OTUs) using abundance-based greedy clustering with a threshold dissimilarity of 0.03 . Singleton OTUs, consisting of a single read across the entire dataset, were culled as unreliable observations. For assessment of phylogenetic diversity and distance measures, Clearcut (Sheneman et al. 2006) was used to generate a rough phylogenetic tree based on one representative sequence per OTU (selected as the most abundant sequence variant within each OTU).

Reads that were culled at the step of matching $16 \mathrm{~S}$ primers were directed through ITSx to identify fungal ITS sequences and trim away flanking regions, including PCR primers. After ITS1 extraction, criteria for processing fungal reads were the same as for bacterial $16 \mathrm{~S}$ reads. The UNITE database v. 7.2 (Kõljalg et al. 2013) was used for classification and chimera identification. Given the well-known challenges of intragenomic variants in ITS sequence, and inability of ITS1 sequences to reliably distinguish among some closely related lineages (Bakker 2018), reads were classified individually and binned into phylotypes by common classification at the species rank rather than clustering into OTUs on a sequence dissimilarity criterion.

In processing taxon abundance tables from amplicon sequencing (one for bacteria and one for fungi), observations from the negative controls were evaluated manually to determine whether component taxa likely represented reagent contamination or sample cross contamination. In the bacterial dataset, 15 OTUs made up $>95 \%$ of the reads in the negative control samples. Of these, 14 OTUs were several times more abundant in the negative controls than in the corresponding biological samples and were manually culled as probable contaminants. This resulted in the removal of approximately $6 \%$ of reads from the biological samples. In the fungal dataset, just three phylotypes made up $>95 \%$ of the reads in the negative controls. Of these, Fusarium and Malassezia spp. appeared likely to be truly present in the biological samples, while the genus Wallemia was manually culled. This resulted in the removal of $<0.001 \%$ of reads from the biological samples. Mock community controls were processed along with the biological samples and were used to estimate error rates in the processed sequence data (Mothur function seq.error). Taxonomic profiles were visualized using Krona (Ondov et al. 2011).

Statistical analyses. Samples were retained for analysis only if they yielded $>2,000$ reads for the bacterial dataset, $>2,500$ reads for the fungal dataset, and had complete data for both toxin content and pathogen load. In total, 100 kernel samples met these criteria.

Taxon observations were rarefied to a common depth $(2,000$ reads for bacterial dataset and 2,500 reads for fungal dataset) (rarefaction curves are provided in Supplementary Fig. S1) ahead of calculating diversity indices. Taxon abundance tables were filtered and transformed ahead of ordination (principal components; function prcomp) and statistical testing. Specifically, taxa present at a mean relative abundance across samples of $<0.1 \%$ of reads and taxa detected in $<15 \%$ of samples were filtered out. Relative abundances among remaining taxa (62 bacterial OTUs and 19 fungal phylotypes) were transformed with the centered log ratio method (Gloor and Reid 2016) with Bayesian multiplicative replacement of zeros (function cmultRelp) (Palarea-Albaladejo and Martin-Fernandez 2015). The significance of fit of metadata onto ordinations was assessed with a permutation test (function envfit) (Oksanen et al. 2018).

Linear models (function $\mathrm{lm}$ ) were used to test relationships between continuous-response variables and putative predictor 
variables. Given that multiple kernels were sometimes collected from the same wheat head, "head nested within year" was used as the first term in all models. Toxin contents (micrograms of DON per gram dry kernel weight) were natural log transformed ahead of modeling. Relative abundance of Fusarium spp. within fungal communities, as observed in amplicon sequencing data, was modeled as centered log ratio transformed values.

Tests for differences in the relative abundance of taxa between categorical groupings of samples were performed using analysis of variance-like differential expression (function $A L D E x$ ) (Gloor and Reid 2016). Random forest regression (function randomForest) (Liaw and Wiener 2002) was used to identify relationships between taxon abundances and pathogen load or DON content.

\section{RESULTS}

Use of a disease screening nursery successfully generated wheat heads manifesting Fusarium head blight symptoms under field conditions. Disease severity (percentage of symptomatic spikelets, assessed for 10 arbitrary heads per plot) in the sampled cultivar was similar between years (mean \pm standard error of $22.1 \pm 3.6$ in 2016 and $25.1 \pm 7.3$ in 2017; data not shown).

Pathogen load, DON content, and dry weight of individual kernels. Pathogen load ranged 40-fold among kernels, was a good predictor of DON content (Pearson correlation $=0.73$ ) (Table 1, model 9; Fig. 1), and did not differ significantly between years $(P=0.41)$. DON was detected in each of the 44 individual kernels that were assessed in 2016 but in only $60 \%$ of kernels from 2017 (34 of 56 kernels). Where present, DON content ranged from DON at 7.3 to $2,197.5 \mu \mathrm{g} \mathrm{g}^{-1}$ dry kernel weight and did not differ significantly among years $(P=0.82$; median where detectable: $35.35 \mu \mathrm{g} \mathrm{g}^{-1}$ in 2016 and $46.70 \mu \mathrm{g} \mathrm{g}^{-1}$ in 2017).
Pathogen load and DON content were each negatively correlated with kernel dry weight (Fig. 1; Supplementary Fig. S2) and were significant predictors of kernel dry weight. However, toxin content explained somewhat more of the variation in kernel weights than did pathogen load (Table 1, models 15 and 16) and pathogen load did not add significant explanatory power to a model that already included toxin content $(P=0.092$; model not shown).

Microbiome profiling: Mock community controls. Inclusion of mock community controls demonstrated successful observation of nearly all expected community members, and accurate classification of reads (see Supplementary Material S2 for expanded discussion of mock community controls).

Microbiome profiling in individual kernels: Summary descriptions. Across 100 individual wheat kernels, our sequencing effort detected 829 bacterial OTUs and 144 fungal phylotypes. Individual, hulled wheat kernels hosted rich and varied microbial communities: 29 to 118 (mean 71.1) bacterial OTUs and 5 to 39 (mean 19.1) fungal phylotypes were detected per kernel. Bacterial OTUs were classified as belonging to nine phyla and 149 named genera, while fungal phylotypes spanned 104 named genera, mainly belonging to phyla Ascomycota and Basidiomycota, with minor contributions from additional phyla (visual summary in Figure 2 and final taxon abundance tables in Supplementary Material S3).

Correlation between the qPCR-based assessment of pathogen load and the amplicon sequencing-based assessment of Fusarium relative abundance was positive and significant (Pearson correlation $=$ $0.63, P<0.001)$. However, the qPCR-based assessment of pathogen load was more effective at explaining kernel DON contents (Table 1, model 9) than was the assessment of Fusarium relative abundance by amplicon sequencing (Table 1, model 8).

Microbiome profiling: Effects of year. Both bacterial and fungal communities on wheat kernels could be clearly differentiated

TABLE 1. Statistical models exploring relationships between pathogen load, deoxynivalenol (DON) content, kernel dry weight, and microbial community diversity indices ${ }^{\mathrm{a}}$

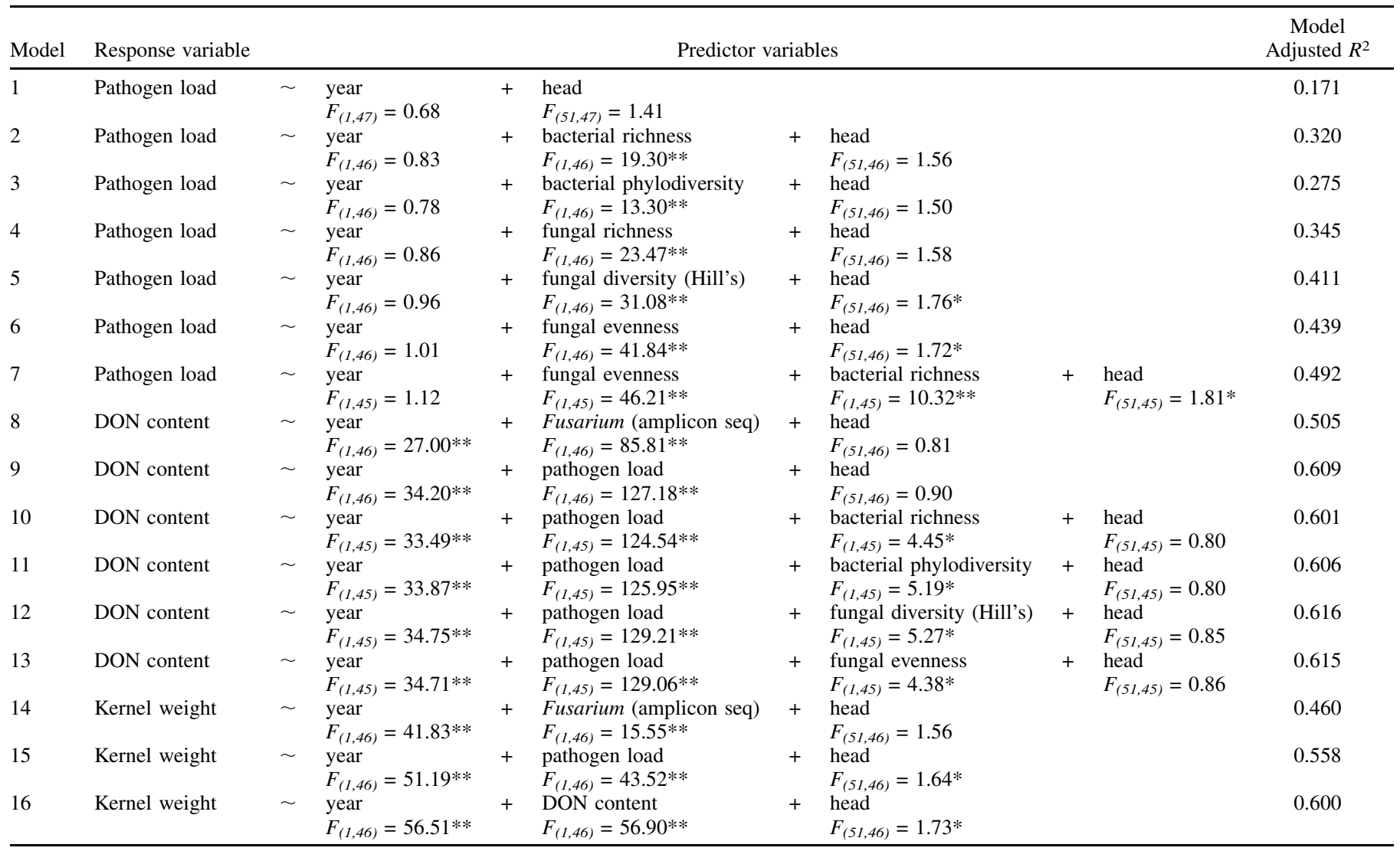

a Asterisks * and $* *$ indicate $P<0.05$ and 0.01 , respectively, and amplicon seq $=$ abundance suggested by amplicon sequence data. 
by year of sampling, with 13 bacterial OTUs and five fungal phylotypes differing significantly in relative abundance among years (Fig. 3; ALDEx; $P<0.01$ ). Observed bacterial community richness and diversity measures did not differ significantly among years (Fig. $4 ; P>0.05$ ). Fungal community diversity was significantly lower in 2016 than in 2017 (Fig. $4 ; P<0.01$ ).

Microbiome profiling: Associations with pathogen load and DON content. Microbial community diversity indices and traits related to Fusarium head blight development (pathogen load, DON content, and seed dry weight) bore significant relationships with bacterial and fungal community structure (Fig. 5; fit to principal components ordination; permutation test, $P<0.05)$.

Measures of bacterial and fungal community diversity were negatively correlated with pathogen load in wheat kernels (Fig. 1). Furthermore, measures of bacterial and fungal community diversity also had significant power to explain pathogen load when included in statistical models that also incorporated head nested within year (Table 1, models 2 to 7).

Random forest regression of pathogen load on bacterial community profiles generated a significant model (37.7\% variance explained; permutation test, $P=0.001$ ), in which two Sphingomonas
OTUs were of dominant importance (Supplementary Fig. S3). For each of these OTUs, as relative abundance within bacterial communities increased, pathogen load decreased (for OTU0017, Pearson correlation $=-0.51, P<0.001$; for OTU0023, Pearson correlation $=-0.54, P<0.001)$. Random forest regression of pathogen load on fungal community profiles similarly generated a significant model (37.7\% variance explained; permutation test, $P=0.001$ ), in which the Fusarium phylotype overshadowed other taxa in importance. In this case, the relationship between taxon abundance and pathogen load was positive, as described above.

Measures of bacterial and fungal community diversity were negatively correlated with kernel DON content (Fig. 1). We expect that the majority of this relationship can be explained via interactions with pathogen load, given that DON is produced by the pathogen. However, there is also a possibility that interactions with other microbes could influence toxin production rates in a manner separate from influences on pathogen density. When added to linear models including head nested within year and pathogen load, measures of bacterial and fungal community diversity were occasionally significant predictors but did not appreciably improve models (Table 1, models 10 to 13). Random forest regressions of detrended DON contents (residuals of the linear model of DON on

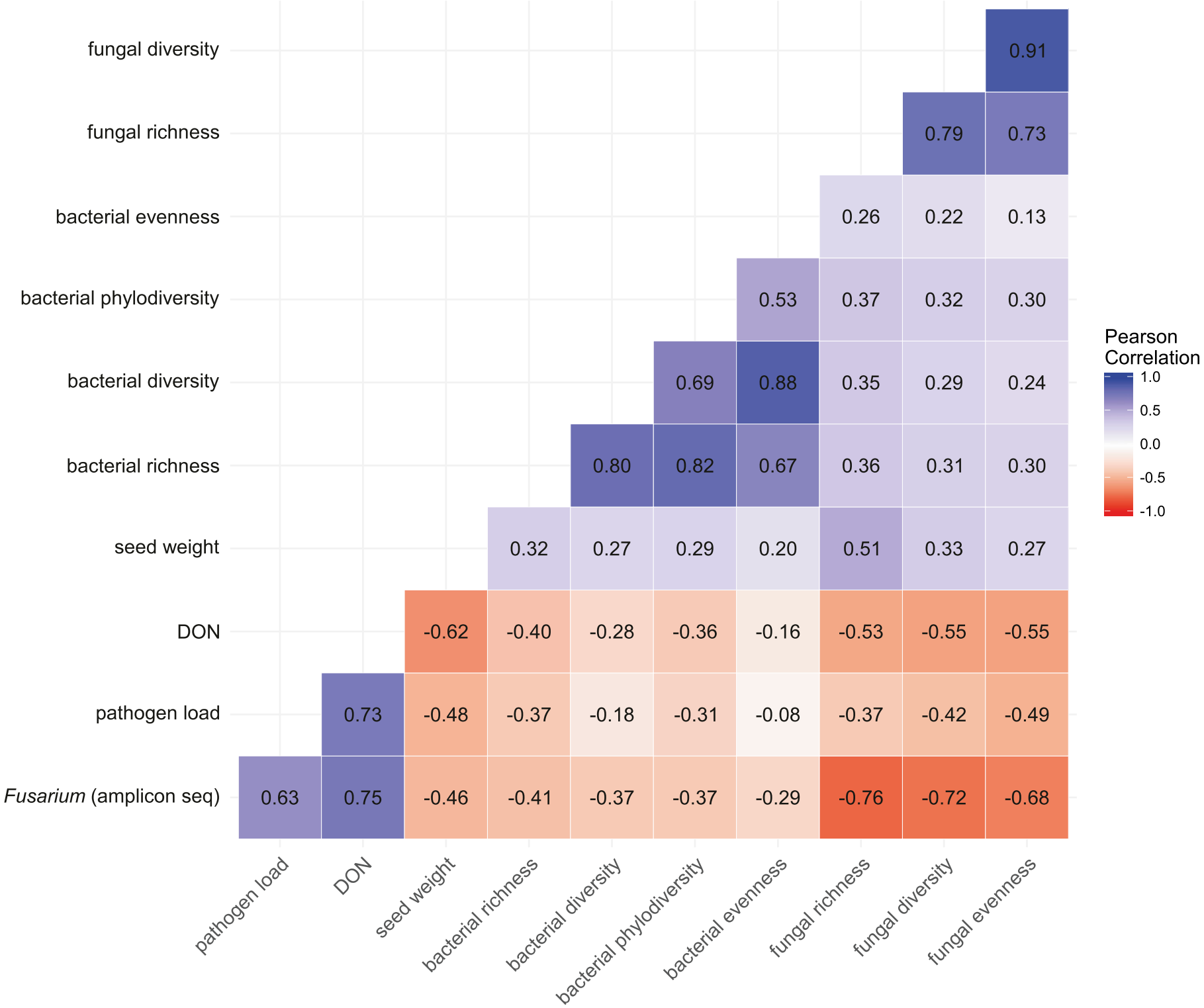

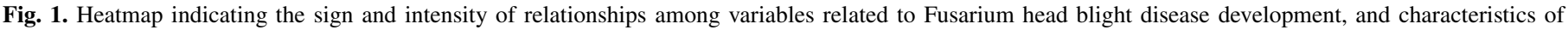
microbiomes associated with wheat kernels. Numbers within the boxes represent Pearson correlation coefficients. DON $=$ deoxynivalenol. 
year/head + pathogen load) on bacterial and fungal community profiles failed to generate significant models, indicating no discernable effect of the microbiome on toxin accumulation that was separate from effects on pathogen load.

\section{DISCUSSION}

We have profiled the bacterial and fungal communities associated with wheat kernels produced in a field environment conducive to Fusarium head blight development. Direct relationships between pathogen biomass, toxin content, and kernel weights are expected and have been described previously (Siou et al. 2014, 2015; Snijders and Perkowski 1990). By relating these measures of pathogen success to microbiome characteristics, we aimed to reveal additional controls on plant disease progression. Follow-up experiments that directly manipulate microbiome characteristics are necessary to test whether any relationships observed here may be causal.

Our data demonstrate a significant ability of microbiome traits to explain variation in pathogen load in wheat kernels, although we are uncertain of the direction of causality. Including the best predictor from among each bacterial and fungal community diversity estimate resulted in an increase in adjusted model $R^{2}$ of 0.321 over a model including as explanatory factors only head nested within year (Table 1, model 7 versus model 1). Improved methods of microbiome profiling may suggest even stronger relationships between microbiome characteristics and functional outcomes. For instance, several of the kernels we sampled exhibited very low bacterial phylogenetic diversity (Fig. 4). In each of these kernels, a large portion of the observed DNA sequences could not be
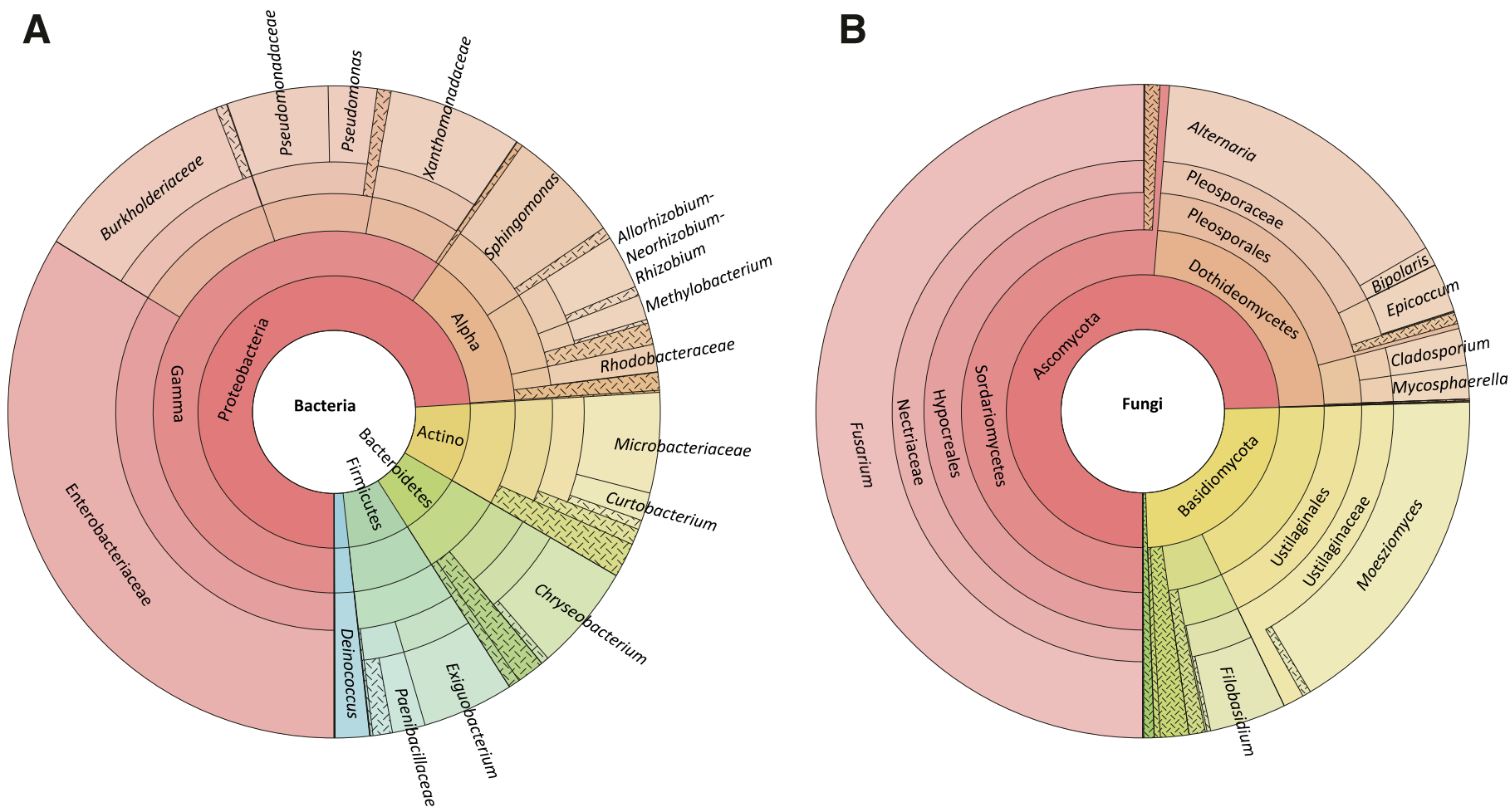

Fig. 2. Visual taxonomic summary of $\mathbf{A}$, bacterial and $\mathbf{B}$, fungal taxa detected on wheat kernels. Different shades represent different taxa, with divisions toward the perimeter reflecting more narrowly defined taxonomic ranks.
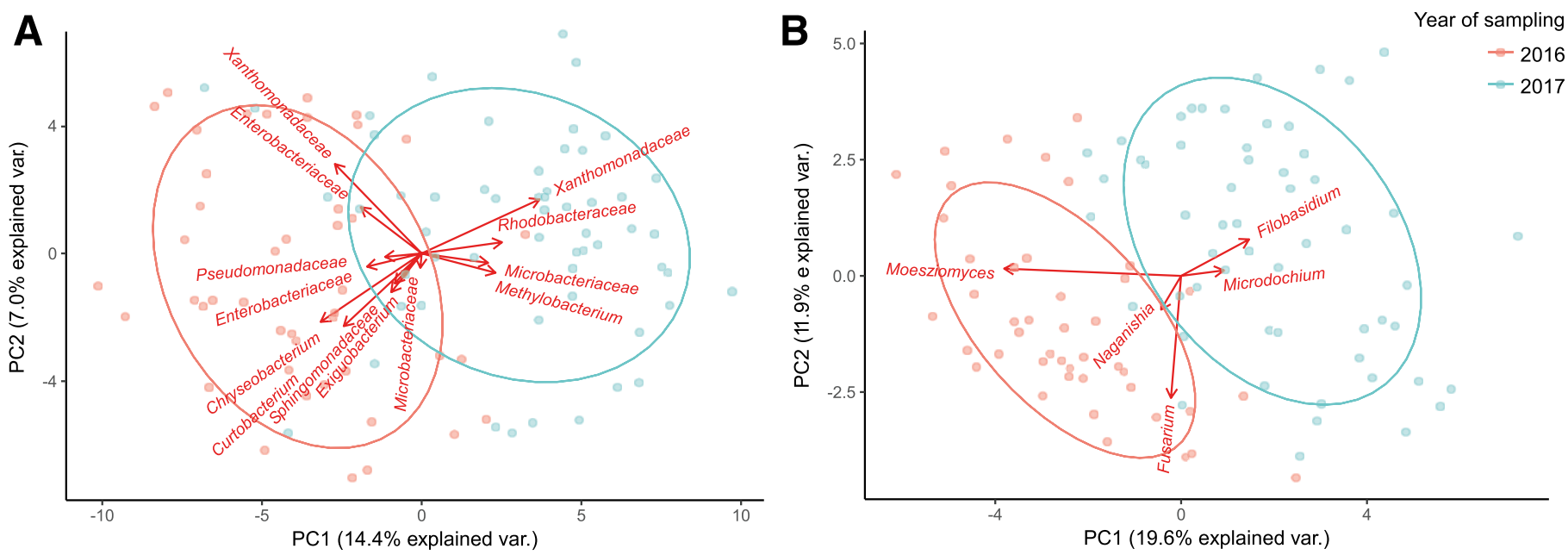

Fig. 3. Principal component (PC) biplots derived from abundance tables of A, bacterial operational taxonomic units and B, fungal phylotypes. Each point represents an observed community from an individual wheat kernel. Points are shaded by year of sampling. Vectors indicate only those taxa whose relative abundance differed significantly between years (ALDEx, $P<0.01$ ). 
confidently placed below the taxonomic rank of family; it is likely that these OTUs encompass multiple taxa among which the particular marker gene fragment we employed is not able to differentiate, and that the true diversity of these samples was higher than our method suggests. It is also possible that some of these OTUs that could not be confidently placed to finely resolved taxonomic ranks may represent residual spurious diversity arising, for example, from sequencing errors.

We deliberately sampled under conditions very favorable to the pathogen in order to ensure that disease developed, and relationships between microbiome characteristics and pathogen success may be stronger under conditions that are less optimal for the pathogen. Management or direct manipulation of microbiome characteristics and taxon abundances may also lead to stronger effects on pathogen success than were observed in this study, in which microbiome assembly on wheat heads was not directly manipulated, aside from introduction of the pathogen.

This work suggests several potential taxa that should be investigated further as potential biological control agents. In particular, several Sphingomonas OTUs showed strong relationships with pathogen load. These relationships may indicate either
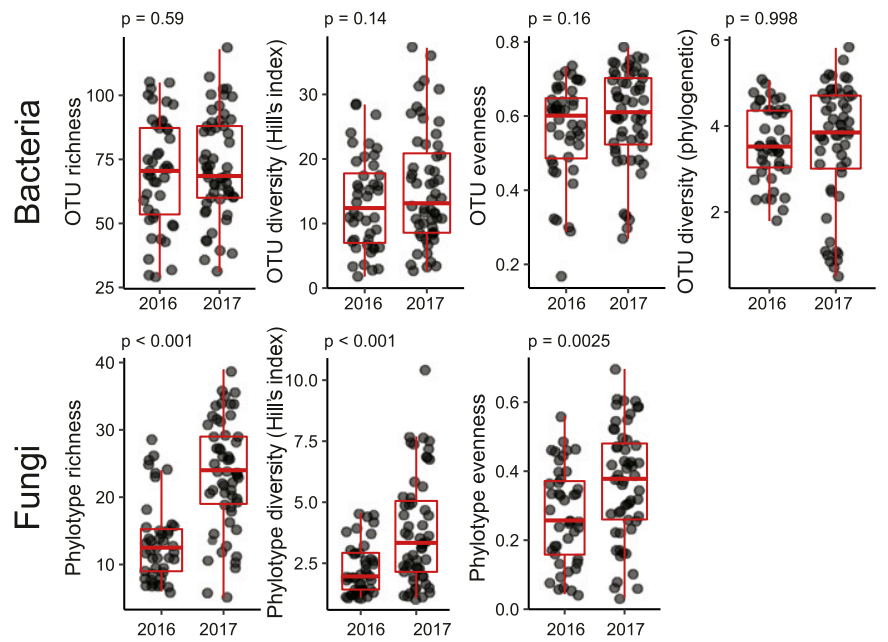

Fig. 4. Comparisons of bacterial and fungal community diversity indices among years for individual wheat kernels. Significance of year as an explanatory factor was assessed using linear models, regressing each diversity index on head nested within year. Boxplots indicate median, first, and third quartiles. Whiskers extend up to 1.5 times the interquartile range. that Sphingomonas spp. are able to inhibit pathogen proliferation or that Sphingomonas spp. decline in abundance as Fusarium spp. proliferate. In a previous report that contrasted culturable bacterial communities from healthy versus Fusarium head blight-infected spikelets, Sphingomonas spp. were sometimes (although not always) recovered at higher proportions from healthy than from diseased spikelets (Yoshida et al. 2012), a pattern which fits our observations. Sphingomonas spp. have been previously investigated as potential biological control agents for protection against wheat diseases, including Fusarium head blight (Wachowska et al. 2013).

Our choice to sample hulled kernels limited the possibility of identifying some mechanisms by which other members of the wheat microbiome may limit Fusarium success. For instance, it is hypothesized that microbial colonists of the anthers may be particularly relevant to limiting initial infection where pathogen entry to the floret is via growth along the anther filament, and one prospective biological control agent for Fusarium head blight was found to colonize wheat anthers inside florets prior to anthesis (Schisler et al. 2014). In contrast, our data address pathogen proliferation within kernels, assuming that the pathogen has already successfully entered the floret.

Observations in this work, as in all other studies that attempt to link microbiome characteristics to functional outcomes, face challenges related to the fine spatial scale at which microbial communities are organized and the rapid temporal dynamics that microbial communities exhibit. For instance, it would have been ideal to characterize microbiomes at an earlier point in time compared with assessment of Fusarium head blight disease parameters. Unfortunately, at present, we are not able to sample the same tissue or observe the same microbiome twice.

Our sampling strategy was spatially targeted and aimed to reduce noise associated with microbiome variation across tissue types in the complex topographic landscape of the wheat head. It has been estimated that a single barley kernel hosts more than 500,000 bacterial cells (Laitila 2007), and aggregate tissue samples such as an entire wheat or barley head represent massive and complex habitats at the microbial scale. Encompassing the wide diversity in this coarse sampling would likely obscure connections between microbiome characteristics and particular components of a given pathosystem. Nevertheless, other recent studies of the microbiome of cereal crops have sampled pooled seed from a common head (Siou et al. 2014) or entire wheat heads (Hertz et al. 2016), or drawn subsamples from larger batches of grain (Links et al. 2014; Nicolaisen et al. 2014).

Our sampling strategy, in which individual kernels were hand picked from intact heads that were produced under very disease-conducive
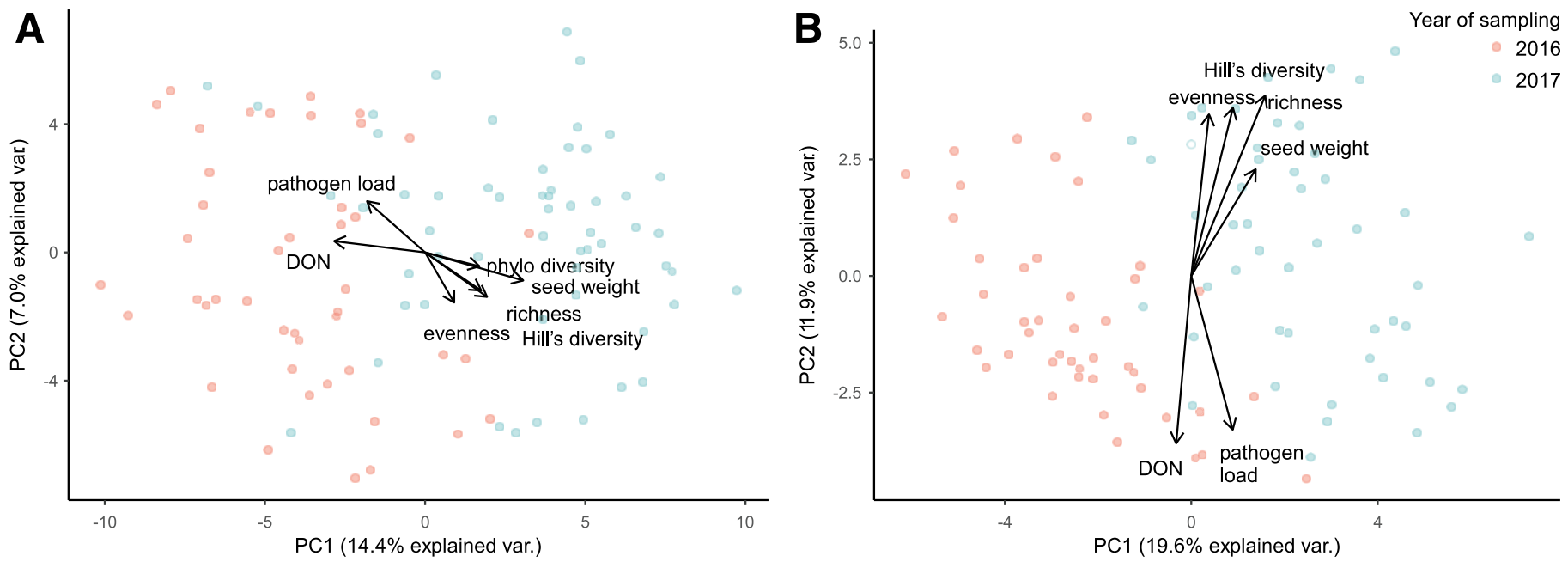

Fig. 5. Principal component $(\mathrm{PC})$ biplots derived from abundance tables of $\mathbf{A}$, bacterial operational taxonomic units and $\mathbf{B}$, fungal phylotypes; each point represents an observed community from an individual kernel. Points are shaded by year of sampling. DON $=$ deoxynivalenol. Vectors reflect associated metadata from the same kernels that have significant fit with the ordination (permutation test, $P<0.05$ ). 
conditions, may also have led to the inclusion of some samples with unusually high toxin contents or pathogen loads. For instance, in one kernel, we observed a toxin content of DON at $>2,000 \mu \mathrm{g} \mathrm{g}^{-1}$ dry kernel weight. Such levels are rarely observed in commercial grain samples, in part because harvesting equipment is typically adjusted to discard the most heavily diseased kernels, which are substantially smaller and lighter than healthy kernels.

The microbiome profiles we observed across our wheat kernels were largely congruent with earlier reports on the composition of bacterial and fungal communities of the phyllosphere of small grains (Gdanetz and Trail 2017; Grudzinska-Sterno et al. 2016; Hertz et al. 2016; Karlsson et al. 2014; Links et al. 2014; Nicolaisen et al. 2014) and, for this reason, are not described in detail. Fungal communities tend to be characterized by a predominance of taxa that include plant pathogens, with the primary exception of some basidiomycete yeasts. However, there is a broad functional range among the constituents of many taxa, and even isolates closely related to plant pathogens can show promise as pathogen antagonists (Gdanetz and Trail 2017). Although unlikely to find use in deliberate biological control, even interaction among two bona fide pathogens can result in reduced disease progression relative to a single pathogen in isolation (Rampitsch et al. 2016; Siou et al. 2015).

We did not attempt to relate abiotic variables to pathogen success or toxin accumulation, because this has been done rigorously as the basis for weather-driven models of Fusarium head blight risk (Del Ponte et al. 2005; De Wolf et al. 2003; Prandini et al. 2009; Shah et al. 2013), and our samples were drawn from an environment that is actively managed to promote disease-conducive conditions. There are also other mechanisms by which some of our measured disease components may have been impacted by effects other than species interactions. For instance, if Fusarium infection progresses into the rachis, resource supply to downstream tissues may be cut off. This could affect kernel weight in a manner largely independent of pathogen load, toxin content, or microbiome characteristics within that kernel.

With the particular measures of pathogen success that we used, there is the possibility of signal amplification in places. For instance, DON may be extracted more efficiently from smaller than from larger kernels, given that we extracted it from intact kernels. This would exaggerate the relationship between toxin content and kernel weight. Similarly, if, at very high densities, saprotrophic activity by Fusarium spp. substantially degrades wheat DNA within infected tissues, our measure of pathogen load would artificially represent very high pathogen loads. Logarithmic transformations of these variables were intended to address signal amplification. Alternative methods carry other own limitations; for instance, expressing pathogen densities on a per-unit-kernel-weight basis ignores the well-known variability in DNA extraction efficiency among samples. Because we only assessed DON content and did not consider other trichothecene variants, there is the possibility that relationships with other variables could have been obscured due to different rates of transformation of DON into modified forms (e.g., formation of DON-glycosides) (Karlovsky 2011) that would have escaped our quantification scheme. It would also be valuable to assess relationships of microbiome characteristics with other mycotoxins (Bakker et al. 2018) such as zearalenone.

A significant correlation was expected between pathogen load, as assessed by qPCR, and Fusarium spp. relative abundance within microbiome profiles. Even so, it is not surprising that the relationship between these variables was weak. It is well known that microbiome profiling by amplicon sequencing is prone to bias and does not accurately reflect absolute abundances (Bakker 2018). The qPCR method was able to detect only Fusarium spp. with the TRI5 gene, the product of which is involved in DON biosynthesis. In contrast, our target locus for amplicon sequencing was not able to differentiate among Fusarium spp. and may have binned together mycotoxigenic and nonmycotoxigenic Fusarium spp. For instance, some kernels without measurable DON content hosted Fusarium spp. within their fungal communities.

Methods for improved taxonomic resolution in identifying the origins of amplicon sequences may have strengthened the relationship between these measures of Fusarium sp. success. However, achieving more detailed taxonomic resolution currently requires trade-offs with breadth of coverage or depth of observation. For instance, longer amplicons allow for differentiation of Fusarium spp. within amplicon sequence data (Walder et al. 2017) but are not compatible with many sequencing platforms, including the MiSeq system used here. Similarly, use of a Fusarium-specific amplicon (Karlsson et al. 2017) allows for differentiation among fusaria but precludes consideration of broader community membership.

There are some challenges in contrasting relationships of Fusarium head blight components with characteristics of bacterial versus fungal communities. Most notably, the pathogen is represented in the fungal dataset but not the bacterial dataset. This makes spurious associations more likely in the fungal dataset, given the compositional nature of the data; we expect large differences in the absolute abundance of Fusarium spp. among seed with different degrees of disease progression, and this change alone could alter the relative abundances of all other fungal taxa. Our data-processing methodology, such as the use of the centered log transformation for the taxon abundance tables, aimed to account for the compositional nature of the data (Gloor and Reid 2016) but may not have completely removed interpretive difficulties.

Our data suggest fairly broad-scale microbiome changes between years, even in the context of a common wheat variety and a heavily managed field setting. This may suggest limits to the potential for microbiome management to effectively enrich desirable taxa. However, interpretation of year-to-year variation in this study is not straightforward, because there is the potential for several confounding factors. These include the separate processing and sequencing of amplicon libraries per year, the use of different numbers of Fusarium strains in the field inoculum, and potentially other factors.

In summary, this observational study highlights microbial species interactions which may affect the development of Fusarium head blight on wheat and the accumulation of trichothecene mycotoxins in grain. Associations suggested in this study should be considered as hypothesis generating, and follow-up studies are required.

\section{ACKNOWLEDGMENTS}

We thank R. Dill-Macky and her team at the University of Minnesota for operating the field trial from which our samples were drawn; and J. Brown, S. Folmar, and A. McGovern for providing excellent technical assistance. Mention of trade names or commercial products is solely for the purpose of providing specific information and does not imply recommendation or endorsement by the U.S. Department of Agriculture (USDA). USDA is an equal opportunity provider and employer. This article was the work of U.S. Government employees engaged in their official duties and is exempt from copyright.

\section{LITERATURE CITED}

Anderson, J. A., Wiersma, J. J., Linkert, G. L., Reynolds, S., Kolmer, J. A., Jin, Y., Dill-Macky, R., and Hareland, G. A. 2015. Registration of 'Rollag' spring wheat. J. Plant Regist. 9:201-207.

Bakker, M. G. 2018. A fungal mock community control for amplicon sequencing experiments. Mol. Ecol. Resour. 18:541-556.

Bakker, M. G., Brown, D. W., Kelly, A. C., Kim, H.-S., Kurtzman, C. P., McCormick, S. P., O’Donnell, K. L., Proctor, R. H., Vaughan, M. M., and Ward, T. J. 2018. Fusarium mycotoxins: A trans-disciplinary overview. Can. J. Plant Pathol. 40:161-171.

Bakker, M. G., Manter, D. K., Sheflin, A. M., Weir, T. L., and Vivanco, J. M. 2012. Harnessing the rhizosphere microbiome through plant breeding and agricultural management. Plant Soil 360:1-13.

Bengtsson-Palme, J., Ryberg, M., Hartmann, M., Branco, S., Wang, Z., Godhe, A., De Wit, P., Sanchez-Garcia, M., Ebersberger, I., de Sousa, F., Amend, A. S., Jumpponen, A., Unterseher, M., Kristiansson, E., Abarenkov, K., 
Bertrand, Y. J. K., Sanli, K., Eriksson, K. M., Vik, U., Veldre, V., and Nilsson, R. H. 2013. Improved software detection and extraction of ITS1 and ITS2 from ribosomal ITS sequences of fungi and other eukaryotes for analysis of environmental sequencing data. Methods Ecol. Evol. 4:914919.

Bulder, A. S., DiNovi, M., Kpodo, K. A., Leblanc, J.-C., Resnik, S., Shephard, G. S., Slob, W., Walker, R., and Wolterink, G. 2011. Pages 317-485 in: Safety Evaluation of Certain Contaminants in Food: Deoxynivalenol. World Health Organization, Geneva, Switzerland.

Chelius, M. K., and Triplett, E. W. 2001. The diversity of archaea and bacteria in association with the roots of Zea mays L. Microb. Ecol. 41:252-263.

Comby, M., Lacoste, S., Baillieul, F., Profizi, C., and Dupont, J. 2016. Spatial and temporal variation of cultivable communities of co-occurring endophytes and pathogens in wheat. Front. Microbiol. 7:403.

Cuperlovic-Culf, M., Vaughan, M. M., Kermillion, K., Surendra, A., Teresi, J., and McCormick, S. P. 2018. Effects of atmospheric $\mathrm{CO}_{2}$ level on the metabolic response of resistant and susceptible wheat to Fusarium graminearum infection. Mol. Plant-Microbe Interact. 32:379-391.

Del Ponte, E. M., Fernandes, J. M. C., and Bergstrom, G. C. 2007. Influence of growth stage on Fusarium head blight and deoxynivalenol production in wheat. J. Phytopathol. 155:577-581.

Del Ponte, E. M., Fernandes, J. M. C., and Pavan, W. 2005. A risk infection simulation model for fusarium head blight of wheat. Fitopatol. Bras. 30: 634-642.

De Wolf, E. D., Madden, L. V., and Lipps, P. E. 2003. Risk assessment models for wheat Fusarium head blight epidemics based on within-season weather data. Phytopathology 93:428-435.

Gao, C.-S., Kou, X.-J., Li, H.-P., Zhang, J.-B., Saad, A. S. I., and Liao, Y.-C. 2013. Inverse effects of Arabidopsis NPR1 gene on fusarium seedling blight and fusarium head blight in transgenic wheat. Plant Pathol. 62:383-392.

Gdanetz, K., and Trail, F. 2017. The wheat microbiome under four management strategies, and potential for endophytes in disease protection. Phytobiomes J. 1:158-168.

Gloor, G. B., and Reid, G. 2016. Compositional analysis: A valid approach to analyze microbiome high-throughput sequencing data. Can. J. Microbiol. 62:692-703.

Grudzinska-Sterno, M., Yuen, J., Stenlid, J., and Djurle, A. 2016. Fungal communities in organically grown winter wheat affected by plant organ and development stage. Eur. J. Plant Pathol. 146:401-417.

Hertz, M., Jensen, I. R., Jensen, L. O., Thomsen, S. N., Winde, J., Dueholm, M. S., Sorensen, L. H., Wollenberg, R. D., Sorensen, H. O., Sondergaard, T. E., and Sorensen, J. L. 2016. The fungal community changes over time in developing wheat heads. Int. J. Food Microbiol. 222:30-39.

Hue, A. G., Voldeng, H. D., Savard, M. E., Fedak, G., Tian, X., and Hsiang, T. 2009. Biological control of Fusarium head blight of wheat with Clonostachys rosea strain ACM941. Can. J. Plant Pathol. 31:169-179.

Jung, B., Park, S. Y., Lee, Y. W., and Lee, J. 2013. Biological efficacy of Streptomyces sp. strain BN1 against the cereal head blight pathogen Fusarium graminearum. Plant Pathol. J. 29:52-58.

Karlovsky, P. 2011. Biological detoxification of the mycotoxin deoxynivalenol and its use in genetically engineered crops and feed additives. Appl. Microbiol. Biotechnol. 91:491-504.

Karlsson, I., Friberg, H., Kolseth, A. K., Steinberg, C., and Persson, P. 2017. Agricultural factors affecting Fusarium communities in wheat kernels. Int. J. Food Microbiol. 252:53-60.

Karlsson, I., Friberg, H., Steinberg, C., and Persson, P. 2014. Fungicide effects on fungal community composition in the wheat phyllosphere. PLoS One 9: e111786.

Kinkel, L. L., Schlatter, D. C., Bakker, M. G., and Arenz, B. E. 2012. Streptomyces competition and co-evolution in relation to plant disease suppression. Res. Microbiol. 163:490-499.

Kõljalg, U., Nilsson, R. H., Abarenkov, K., Tedersoo, L., Taylor, A. F. S., Bahram, M., Bates, S. T., Bruns, T. D., Bengtsson-Palme, J., Callaghan, T. M., Douglas, B., Drenkhan, T., Eberhardt, U., Dueñas, M., Grebenc, T., Griffith, G. W., Hartmann, M., Kirk, P. M., Kohout, P., Larsson, E., Lindahl, B. D., Lücking, R., Martín, M. P., Matheny, P. B., Nguyen, N. H., Niskanen, T., Oja, J., Peay, K. G., Peintner, U., Peterson, M., Põldmaa, K., Saag, L., Saar, I., Schüßler, A., Scott, J. A., Senés, C., Smith, M. E., Suija, A., Taylor, D. L., Telleria, M. T., Weiss, M., and Larsson, K. H. 2013. Towards a unified paradigm for sequence based identification of fungi. Mol. Ecol. 22: 5271-5277.

Laitila, A. 2007. Microbes in the Tailoring of Barley Malt Properties. University of Helsinki, Helsinki, Finland.

Larran, S., Perelló, A., Simón, M. R., and Moreno, V. 2002. Isolation and analysis of endophytic microorganisms in wheat (Triticum aestivum L.) leaves. World J. Microbiol. Biotechnol. 18:683-686.

Larran, S., Perelló, A., Simón, M. R., and Moreno, V. 2007. The endophytic fungi from wheat (Triticum aestivum L.). World J. Microbiol. Biotechnol. 23:565-572.
Legard, D. E., McQuilken, M. P., Whipps, J. M., Fenlon, J. S., Fermor, T. R., Thompson, I. P., Bailey, M. J., and Lynch, J. M. 1994. Studies of seasonal changes in the microbial populations on the phyllosphere of spring wheat as a prelude to the release of a genetically modified microorganism. Agric. Ecosyst. Environ. 50:87-101.

Legrand, F., Picot, A., Cobo-Díaz, J. F., Chen, W., and Le Floch, G. 2017. Challenges facing the biological control strategies for the management of Fusarium head blight of cereals caused by $F$. graminearum. Biol. Control 113:26-38.

Liaw, A., and Wiener, M. 2002. Classification and regression by randomForest. R News 2/3:18-22.

Links, M. G., Demeke, T., Grafenhan, T., Hill, J. E., Hemmingsen, S. M., and Dumonceaux, T. J. 2014. Simultaneous profiling of seed-associated bacteria and fungi reveals antagonistic interactions between microorganisms within a shared epiphytic microbiome on Triticum and Brassica seeds. New Phytol. 202:542-553.

Lundberg, D. S., Lebeis, S. L., Paredes, S. H., Yourstone, S., Gehring, J., Malfatti, S., Tremblay, J., Engelbrektson, A., Kunin, V., Rio, T. G., Edgar, R. C., Eickhorst, T., Ley, R. E., Hugenholtz, P., Tringe, S. G., and Dangl, J. L. 2012. Defining the core Arabidopsis thaliana root microbiome. Nature 488:86-90.

Nganje, W. E., Johnson, D. D., Wilson, W. W., Leistritz, F. L., Bangsund, D. A., and Tiapo, N. M. 2001. Economic impacts of Fusarium head blight in wheat and barley: 1998-2000. Agribusiness and Applied Economics Rep. No. 464. North Dakota State University, Fargo, ND, U.S.A.

Nicolaisen, M., Justesen, A. F., Knorr, K., Wang, J., and Pinnschmidt, H. O. 2014. Fungal communities in wheat grain show significant co-existence patterns among species. Fungal Ecol. 11:145-153.

Oksanen, J., Blanchet, F. G., Friendly, M., Kindt, R., Legendre, P., McGlinn, D., Minchin, P. R., O'Hara, R. B., Simpson, G. L., Solymos, P., Stevens, M. H. H., Szoecs, E., and Wagner, H. 2018. vegan: Community ecology package. R package version 2.5-1. https://cran.r-project.org/web/packages/ vegan/index.html

Ondov, B. D., Bergman, N. H., and Phillippy, A. M. 2011. Interactive metagenomic visualization in a web browser. BMC Bioinf. 12:385.

Palarea-Albaladejo, J., and Martin-Fernandez, J. A. 2015. zCompositions-R package for multivariate imputation of left-censored data under a compositional approach. Chemom. Intell. Lab. Syst. 143:85-96.

Prandini, A., Sigolo, S., Filippi, L., Battilani, P., and Piva, G. 2009. Review of predictive models for Fusarium head blight and related mycotoxin contamination in wheat. Food Chem. Toxicol. 47:927-931.

Quast, C., Pruesse, E., Yilmaz, P., Gerken, J., Schweer, T., Yarza, P., Peplies, J., and Glockner, F. O. 2013. The SILVA ribosomal RNA gene database project: Improved data processing and web-based tools. Nucleic Acids Res. 41:D590-D596.

Ramakers, C., Ruijter, J. M., Deprez, R. H. L., and Moorman, A. F. M. 2003. Assumption-free analysis of quantitative real-time polymerase chain reaction (PCR) data. Neurosci. Lett. 339:62-66.

Rampitsch, C., Bacala, R., Tekauz, A., and McCallum, B. D. 2016. Inhibition of Fusarium graminearum and other Fusarium species by Cochliobolus sativus in culture and on barley plants. Can. J. Plant Pathol. 38:422-429.

R Core Team. 2015. R: A Language and Environment for Statistical Computing. R Foundation for Statistical Computing, Vienna, Austria.

Rognes, T., Flouri, T., Nichols, B., Quince, C., and Mahe, F. 2016. VSEARCH: A versatile open source tool for metagenomics. PeerJ 4:e2584.

Ruijter, J. M., Ramakers, C., Hoogaars, W. M., Karlen, Y., Bakker, O., van den Hoff, M. J., and Moorman, A. F. 2009. Amplification efficiency: Linking baseline and bias in the analysis of quantitative PCR data. Nucleic Acids Res. 37:e45.

Schisler, D. A., Boehm, M. J., Paul, P. A., Rooney, A. P., and Dunlap, C. A. 2015. Reduction of Fusarium head blight using prothioconazole and prothioconazole-tolerant variants of the Fusarium head blight antagonist Cryptococcus flavescens OH 182.9. Biol. Control 86:36-45.

Schisler, D. A., Core, A. B., Boehm, M. J., Horst, L., Krause, C., Dunlap, C. A., and Rooney, A. P. 2014. Population dynamics of the Fusarium head blight biocontrol agent Cryptococcus flavescens $\mathrm{OH} 182.9$ on wheat anthers and heads. Biol. Control 70:17-27.

Schloss, P. D., Westcott, S. L., Ryabin, T., Hall, J. R., Hartmann, M., Hollister, E. B., Lesniewski, R. A., Oakley, B. B., Parks, D. H., Robinson, C. J., Sahl, J. W., Stres, B., Thallinger, G. G., Horn, D. J. V., and Weber, C. F. 2009. Introducing mothur: Open-source, platform-independent, communitysupported software for describing and comparing microbial communities. Appl. Environ. Microbiol. 75:7537-7541.

Shah, D. A., Molineros, J. E., Paul, P. A., Willyerd, K. T., Madden, L. V., and De Wolf, E. D. 2013. Predicting Fusarium head blight epidemics with weather-driven pre- and post-anthesis logistic regression models. Phytopathology 103:906-919.

Sheneman, L., Evans, J., and Foster, J. A. 2006. Clearcut: A fast implementation of relaxed neighbor joining. Bioinformatics 22:2823-2824. 
Simpson, D. R., Weston, G. E., Turner, J. A., Jennings, P., and Nicholson, P. 2001. Differential control of head blight pathogens of wheat by fungicides and consequences for mycotoxin contamination of grain. Eur. J. Plant Pathol. 107:421-431.

Siou, D., Gelisse, S., Laval, V., Elbelt, S., Repincay, C., Bourdat-Deschamps, M., Suffert, F., and Lannou, C. 2015. Interactions between head blight pathogens: Consequences for disease development and toxin production in wheat spikes. Appl. Environ. Microbiol. 81:957-965.

Siou, D., Gélisse, S., Laval, V., Repinçay, C., Canalès, R., Suffert, F., and Lannou, C. 2014. Effect of wheat spike infection timing on fusarium head blight development and mycotoxin accumulation. Plant Pathol. 63:390-399.

Smith, D. P., and Peay, K. G. 2014. Sequence depth, not PCR replication, improves ecological inference from next generation DNA sequencing. PLoS One 9:e90234.

Snijders, C. H. A., and Perkowski, J. 1990. Effects of head blight caused by Fusarium culmorum on toxin content and weight of wheat kernels. Phytopathology 80:566-570.

Starkey, D. E., Ward, T. J., Aoki, T., Gale, L. R., Kistler, H. C., Geiser, D. M., Suga, H., Tóth, B., Varga, J., and O’Donnell, K. 2007. Global molecular surveillance reveals novel Fusarium head blight species and trichothecene toxin diversity. Fungal Genet. Biol. 44:1191-1204.
Wachowska, U., Irzykowski, W., Jedryczka, M., Stasiulewicz-Paluch, A. D., and Głowacka, K. 2013. Biological control of winter wheat pathogens with the use of antagonistic Sphingomonas bacteria under greenhouse conditions. Biocontrol Sci. Technol. 23:1110-1122.

Walder, F., Schlaeppi, K., Wittwer, R., Held, A. Y., Vogelgsang, S., and van der Heijden, M. G. A. 2017. Community profiling of Fusarium in combination with other plant-associated fungi in different crop species using SMRT sequencing. Front. Plant Sci. 8:2019.

Wang, Q., Garrity, G. M., Tiedje, J. M., and Cole, J. R. 2007. Naïve Bayesian classifier for rapid assignment of rRNA sequences into the new bacterial taxonomy. Appl. Environ. Microbiol. 73:5261-5267.

Windels, C. E. 2000. Economic and social impacts of Fusarium head blight: Changing farms and rural communities in the Northern Great Plains. Phytopathology 90:17-21.

Windels, C. E., Burnes, P. M., and Kommedahl, T. 1993. Fusarium species stored on silica gel and soil for ten years. Mycologia 85:21-23.

Yoshida, S., Ohba, A., Liang, Y. M., Koitabashi, M., and Tsushima, S. 2012. Specificity of Pseudomonas isolates on healthy and Fusarium head blight-infected spikelets of wheat heads. Microb. Ecol. 64:214225 . 